\title{
Work Engagement y Compromiso Laboral: ¿Es la edad un factor diferenciador?
}

\author{
Lina Marcela Guevara Bedoya' ${ }^{7}$ Luisa Fernanda Fernández Poveda²
}

Recibido: 21/09/2016 Aceptado: 24/04/2017

DOI: $10.21772 /$ ripo.v34n2a04

\begin{abstract}
Resumen
En este artículo se presenta el análisis comparativo por edades de los tres componentes del Compromiso Laboral de acuerdo con el modelo de Meyer y Allen (1997), más la variable Work Engagement conceptualizada por Schaufeli y Salanova (2009). Se trabajó con una muestra de 17.165 empleados de empresas latinas de los cuales el $92 \%$ eran colombianos. La muestra cubrió los sectores económicos de servicios (55\%) industrial (27\%), comercial, educativo y de salud (18\%). El 78\% no tenía personal a cargo y el $62 \%$ era de género masculino. Luego de analizar la distribución de los datos con KolmogorovSmirnov se optó por el uso de pruebas estadísticas no paramétricas para el análisis de los mismos (Kruskal Wallis y U de Mann-Whitney). Se encontraron diferencias significativas en la mayoría de comparaciones establecidas $(\mathrm{p}<0.05)$ indicando que a mayor edad mayor fuerza del vínculo, sin embargo el efecto fue pequeño $(\mathrm{d}<0.2)$. Se concluye que la edad sí es un factor diferenciador en las variables estudiadas aunque con bajo impacto.
\end{abstract}

Palabras clave: Compromiso Organizacional, Work Engagement, Edad.

\section{Work Engagement and Organizational Commitment: Is age a differentiator factor?}

\begin{abstract}
This article presents the comparative analysis by age of the three components of the Organizational Commitment according to Meyer and Allen's (1997) model, plus the variable Work Engagement conceptualized by Schaufeli and Salanova (2009). This work was done on a sample of 17165 employees from Latin American organization from which $92 \%$ were Colombians. The sample covered the services (55\%), industrial (27\%), comercial, academic and health (18\%) economic sectors. $78 \%$ of the sample didn't have staff and $62 \%$ were of male gender. After analyzing the distribution of the data with Kolmogorov-Smirnov it was decided the use of non-parametric statistical tests for data analysis (Kruskal Wallis y U de Mann-Whitney). Significant differences were found in most of the established comparisons $(p<0.05)$ showing evidence that to higher ages higher the strength of the bond, however, the effect was small. $(\mathrm{d}<0.2)$. It's concluded that age is indeed a differentiating factor on the studied variables though its impact on them is low.
\end{abstract}

Keywords: Organizational Commitment, Work Engagement, Age.

Psicóloga. Especialista en Gerencia del Desarrollo Humano. Universidad EAFIT. Medellín. Colombia. E-mail: lguevara@cincel.com.co Psicóloga. Especialista en Psicología del Trabajo y de las Organizaciones. Universidad de San Buenaventura. Medellín. Colombia. E-mail: Ifernandez@ cincel.com.co

CINCEL S.A.S.: Centro de Investigación en Comportamiento Organizacional cuyo equipo se dedica a las labores de consultoría y de investigación científica sobre los asuntos humanos del trabajo en organizaciones de Colombia y de América Latina. Para mayor información visitar http://cincel.com.co/

Cómo citar este artículo: Fernández Poveda, L. F., \& Guevara Bedoya, L. M. (2015). Work Engagement y Compromiso Laboral: ¿Es la edad un factor diferenciador? Revista Interamericana de Psicología Ocupacional, 34(2), 108-119. Doi: 10.21772/ripo.v34n2a04 


\section{Introducción}

¿Existe realmente una relación entre la edad y el compromiso laboral? ¿Cuáles son las formas en las cuales las diferentes generaciones se vinculan con la organización? ¿Qué apasiona a las personas de las organizaciones? ¿Qué hacer para que la gente joven permanezca en la empresa? Estas y otras inquietudes, sumadas a la creciente queja del personal de recursos humanos sobre el aumento en los costos por la rotación de los más jóvenes, han despertado el interés por estudiar rigurosamente los datos recolectados en CINCEL S.A.S. ${ }^{1}$ y la información científica disponible sobre Compromiso Laboral y Work Engagement. Este artículo reúne la información recolectada durante tres años consecutivos y busca dar respuesta a si realmente existen variaciones en los componentes del Compromiso Laboral y del Work Engagement en función de la edad. Adicionalmente se plantean algunas hipótesis, basadas en la revisión de otras investigaciones, respecto a los factores que podrían explicar las diferencias encontradas.

\section{Compromiso Laboral y Work Engagement}

El Compromiso Laboral se define teóricamente como la fuerza relativa de la identificación y el involucramiento del individuo con una organización. Es el vínculo psicológico que une a la persona con el lugar en el cual trabaja. La literatura señala tres características del compromiso: la aceptación y creencia en las metas y los valores propios de la organización, la voluntad de esforzarse por alcanzar los logros de la compañía, y la intención de permanecer en la misma (Mowday, Steers, \& Porter, 1979). Estas tres características pueden o no presentarse conjuntamente.

Existen a su vez tres componentes que determinan el Compromiso Laboral y que pueden presentarse de forma simultánea, aunque

1 CINCEL S.A.S.: Centro de Investigación en Comportamiento Organizacional cuyo equipo se dedica a las labores de consultoría y de investigación científica sobre los asuntos humanos del trabajo en organizaciones de Colombia y de América Latina. Para mayor información visitar http://cincel.com.co/ en diferentes proporciones. El Compromiso Afectivo, el Normativo y el de Continuidad (Allen \& Meyer, 1990). El primero es la disposición emocional positiva del empleado hacia la empresa, por la que se identifica, involucra y disfruta de la pertenencia a ella. El segundo, la disposición cognitivo-afectiva del empleado hacia la empresa, por la que permanece y se involucra de acuerdo con consideraciones de gratitud, reciprocidad y deber moral. El tercero, la disposición racional del empleado hacia la empresa, por la que permanece y se involucra de acuerdo con sus estimaciones de costos y beneficios, no necesariamente porque le interese su trabajo $\mathrm{u}$ organización (Meyer, Stanley, Herscovitch \& Topolnytsky, 2002). Múltiples investigaciones han evidenciado que el Compromiso de Continuidad solamente tiene efectos sobre la intención de permanecer vinculado y no suele incidir positivamente en el desempeño (Littlewood, 2003; Mathieu \& Zajac, 1990; Meyer 2014; Meyer et al, 2002).

Lo señalado anteriormente corresponde a las formas de comprometerse con la organización. Existe también una forma particular de relacionarse con el trabajo o la tarea, que se denomina Work Engagement (Salanova, 2009). Este fenómeno se define como un estado psicológico positivo afectivo caracterizado por el vigor, la dedicación y la absorción. El primero se refiere a altos niveles de energía, de activación o de resistencia; la dedicación da cuenta de altos niveles de entusiasmo, inspiración y orgullo por la actividad, y la absorción significa altos niveles de concentración y se experimenta como la sensación de que "el tiempo se pasa volando" (Salanova, Schaufeli, Llorens, Peiró \& Grau, 2000). Es importante considerar que una persona puede estar comprometida con su tarea (Engage) y no con la organización, de igual manera, podría estar comprometida con la organización sin que se encuentre apasionada por su trabajo. Pese a ello, diversas investigaciones han reportado relaciones entre Work Engagement y Compromiso Laboral (Castrejón, 2016; Meyer, 2014) en tanto ambos son determinantes importantes de la acción. 
Es importante aclarar que, pese a que se encuentran investigaciones recientes que tratan los conceptos de Work Engagement y Compromiso Laboral como un solo constructo (López, García \& Pando 2015), estas dos variables corresponden a modelos conceptuales diferentes. En esta investigación se abordan ambas dado que comparten su sustento en las teorías motivacionales (Almeida \& Fierro, 2016; Meyer, 2014; Zager, 2015) y solo con la consideración de las dos era posible explorar la dimensión relacionada con el trabajo y la que concierne a la organización.

\section{Generaciones en el contexto laboral}

Las diversas variaciones en los niveles de Compromiso Laboral y Work Engagement han tratado de entenderse a partir de distintas explicaciones, siendo una de ellas la que alude al cambio generacional (Simon \& Allard, 2007). Sin embargo, se hace necesario llevar a cabo procesos investigativos que permitan validarlas desde una perspectiva rigurosa y en el contexto latinoamericano. A ello se añade el tema de diversidad e inclusión que se ha tornado como un reto para las áreas de gestión de recursos humanos en tanto están en el deber de generar estrategias que integren de forma eficiente el trabajo de personas con capacidades e intereses diferentes (Calderón, Naranjo \& Álvarez, 2010).

Un estudio realizado en Latinoamérica señala que el mercado laboral acoge trabajadores de cuatro generaciones que cubren un rango de más de 40 años de edad (Chirinos, 2009). En investigaciones norteamericanas se ha reportado que desde hace algunos años interactúan cuatro generaciones en el mismo contexto laboral (Irizarry \& de Arecibo, 2009; Lowe, Levitt, \& Wilson, 2011). Es posible encontrar jóvenes de 18 y adultos de más de 60 años trabajando en una misma empresa e incluso en un mismo equipo.

Las etapas del desarrollo relacionadas con la formación académica, el vínculo matrimonial y la procreación se hallan menos delimitadas que hace algunas décadas (Valdivieso, 2002), por tanto, estudiar al ser humano desde la perspectiva evolutiva debe hacerse hoy de manera diferente. Actualmente las etapas se superponen lo que hace difícil establecer consenso en torno a los años que podrían marcar la diferencia entre una generación y otra (Irizarry \& de Arecibo, 2009), esto pese a que, por asuntos metodológicos, se establezcan rangos para diferenciar los grupos generacionales.

Los estudios que contemplan la edad como una variable diferenciadora suelen recurrir al concepto de generación. Dicho término alude a un grupo de personas que han atravesado por procesos de aprendizaje o culturales similares, dependientes de haber nacido en un lapso definido de tiempo (Ogg \& Bonvalet, 2006). Estudiar fenómenos psicosociales a partir de cohortes generacionales es una práctica antigua y obedece al reconocimiento de que el contenido cambiante de la educación formal, las diferentes formas de socialización y el contexto político en el que se desenvuelve un grupo de individuos, genera semejanzas entre quienes se enfrentan simultáneamente a estos hechos (Ryder, 1965).

Las preferencias laborales de los diferentes grupos generacionales varían por las diversas visiones que tienen del mundo (Park, \& Gursoy, 2012), lo que obliga a las organizaciones a buscar e implementar estrategias de atracción y retención del personal para lograr la continuidad de las nuevas generaciones.

Dado todo lo expuesto anteriormente el objetivo de esta investigación fue determinar si la edad es un factor diferenciador en los niveles de Work Engagement y de los distintos componentes del Compromiso Laboral. Se tuvo como hipótesis que las personas más jóvenes presentaban los niveles más bajos en las variables señaladas. Vale mencionar que no se encontraron antecedentes investigativos similares en el contexto Colombiano con una muestra suficientemente representativa como la del presente estudio, por lo que este se considera novedoso y aportante. 


\section{Método}

Este es un estudio descriptivo comparativo de corte transversal que recopila los datos recogidos durante tres años $(2013,2014$ y 2015). Todos los datos provienen de estudios realizados por el Centro de Investigación en Comportamiento Organizacional CINCEL S.A.S. a empresas que contrataron el servicio diagnóstico del Compromiso Laboral y Work Engagement.

\section{Procedimiento}

Para cada estudio los participantes de la empresa llenaron una encuesta anónima previo diligenciamiento del consentimiento informado en el que se señalaba que los datos podrían ser usados para investigación y que las empresas solo recibirían reportes por grupos de más de cinco personas.

Ambas encuestas tardaban un promedio de 20 minutos en ser contestadas. El $52 \%$ de los datos fue recopilado de manera presencial estando las aplicaciones a cargo de profesionales del centro en grupos de máximo 25 personas, el porcentaje restante se capturó a través de la plataforma virtual Survey Monkey. Las diferencias según el tipo de aplicación fueron significativas a nivel estadístico siendo superiores en las aplicaciones presenciales $(\mathrm{p}<0.001)$ en todas las variables analizadas (Work Engagement $\mathrm{t}=32.2$ Compromiso Afectivo $\mathrm{t}=26.9$ Compromiso Normativo $t=22.3$ Compromiso de continuidad $\mathrm{t}=5.4$ ) sin embargo, dado que el personal que contestaba online era administrativo y el restante operativo, no es posible saber si las diferencias obedecían a la forma de aplicar la encuesta o a otras características del personal.

\section{Participantes}

Se trabajó con una muestra de 17.165 casos de empleados con contrato laboral a término indefinido (83\%) y contrato a término fijo provenientes de 36 medianas y grandes empresas de diferentes países, aunque con un marcado sesgo colombiano ( $92 \%$ de los datos). El 8\% restante se distribuye entre México,
Costa Rica, Perú, Panamá, Chile y Guatemala. La muestra cubrió los sectores económicos de servicios (55\%) industrial (27\%), comercial, educativo y de salud (18\%). Las diferencias por sectores fueron estadísticamente significativas en todas las variables $(p<0.01)$ siendo ligeramente mayores todas las puntuaciones en las empresas del sector industrial y menores en el sector educativo y de salud (Work Engagement $\mathrm{F}=353.27$, Compromiso Afectivo $\mathrm{F}=$ 168.60, Compromiso de Continuidad $\mathrm{F}=7.78$, Compromiso Normativo $\mathrm{F}=148.99$, $\mathrm{gl}=4$ ). El $78 \%$ no tenía personal a cargo y el $62 \%$ era de género masculino. El grupo se dividió en cuatro rangos de edad así: De 18 a 25 años $(\mathrm{N}=1594)$, de 26 a 35 años $(\mathrm{N}=5516)$, de 36 a 45 años $(\mathrm{N}=3752)$ y 46 años o más $(\mathrm{N}=6303)$. Todas las personas contaban con capacidad de lecto-escritura para diligenciar la encuesta de forma individual.

La muestra fue tomada por disponibilidad de las empresas clientes de CINCEL S.A.S., por lo tanto fueron estas quienes definían los distintos grupos de edad. Para la presente investigación se homologaron los rangos buscando conservar la mayor cantidad de datos posibles. De allí surgió la agrupación anteriormente señalada.

\section{Instrumentos}

Los instrumentos empleados en este estudio fueron diseñados y validados por el Centro de Investigación en Comportamiento Organizacional CINCEL S.A.S. quien facilitó tanto los cuestionarios como las fichas técnicas para proceder con el análisis.

Nexos. Escala de Compromiso Laboral y Work Engagement. Se divide en dos partes: la primera consta de diez ítems con los que se evalúa Work Engagement con afirmaciones como "El tiempo $<<$ vuela >> cuando estoy trabajando" a los que se debe responder en una escala de frecuencia que va de (5) Siempre, a (1) Nunca.

La segunda parte, que evalúa los tres componentes de Compromiso, se subdivide en tres 
bloques que evalúan las razones por las cuales "vale la pena esforzarse en el trabajo", "hay identificación con las normas, valores y objetivos de la empresa", y "no vale la pena retirarse de la empresa para trabajar en otra" (cada una de estas frases es el encabezado de un bloque). Vale aclarar que el instrumento no reporta resultados por bloques sino por componentes del constructo. Los dos primeros bloques contienen razones que evalúan los componentes afectivo y normativo. El último contiene los tres componentes. Compromiso Afectivo contiene ocho ítems con frases como "Me siento orgulloso(a) de mi empresa". Compromiso Normativo tiene seis ítems entre los que se encuentra "Le debo mucho a esta empresa ". Compromiso de Continuidad tiene cuatro ítems de afirmaciones como "Recibo un mejor salario aquí del que recibiría en otra empresa”. Todas las frases se presentan luego del enunciado que encabeza cada bloque. El cuestionario se responde con una escala tipo Likert que va de (0) no estoy seguro del asunto, a (4) totalmente de acuerdo. El cuestionario total tiene un nivel de confiabilidad de 0.91 y las subescalas varían entre 0.78 y 0.90 (CINCEL S.A.S., 2015).

Se trabajó adicionalmente con un cuestionario de datos sociodemográficos que contenía variables como género y edad, ambas medidas de forma categórica.

\section{Análisis de datos}

Los datos se consolidaron en un solo archivo. Primero se verificó la calidad psicométrica de los instrumentos puesto que, pese a que se contaba con las fichas técnicas con los datos de validación de ambos, era necesario verificar que eran adecuados para usarlos en la muestra seleccionada. Los datos de confiabilidad se reportan en la tabla 1 en el apartado de resultados.

Luego se calcularon los resultados en puntuación directa para cada una de las variables del estudio. Se obtuvieron datos descriptivos de media y desviación estándar, y de correlaciones con el método de
Spearman. Se consideran correlaciones moderadas aquellas que sean significativas $(p<0.01)$ y cuyos valores oscilen entre 0.3 y 0.6 . Posteriormente se analizó la distribución de los datos con la prueba de Kolmogorov-Smirnov para determinar qué tipo de estadísticos debían usarse. Se asume que la distribución es no paramétrica cuando se rechaza la hipótesis de normalidad $(p<0.01)$. Se utilizó la prueba de Kruskal Wallis para establecer si la edad como criterio de comparación entre grupos explicaba las diferencias estadísticamente significativas en las medidas de Compromiso Laboral y Work Engagement. Se establecieron todas las comparaciones posibles entre pares de grupos y se utilizó la prueba $\mathrm{U}$ de Mann-Whitney para reducir el error en el análisis e identificar de forma más específica dónde se producían las diferencias. Para todo ello se utilizó el SPSS 22. Finalmente, en Excel, se utilizó la $d$ de Cohen para calcular el tamaño del efecto. De acuerdo con este estadístico, un tamaño del efecto de 0.2 a 0.3 se considera pequeño, en torno a 0.5 se califica como medio y mayores a este pueden asumirse como grandes (Coe \& Merino, 2003).

\section{Consideraciones éticas}

La propuesta de investigación fue estudiada y avalada por el Comité Científico de CINCEL S.A.S. quien revisó las implicaciones a la luz de la ley 1090 de 2006 y el código deontológico y de bioética de la psicología en Colombia.

Las empresas recibieron resultados generales y por grupos de cinco personas o más. En ningún momento se reportaron datos individuales o que permitiesen identificar las respuestas de cada uno de los encuestados.

\section{Resultados}

En la Tabla 1 pueden observarse los datos correspondientes al nivel de confiabilidad de cada una de las escalas $(\alpha)$ y los datos descriptivos de Mínimo, Máximo, Media y Desviación Estándar. También se encuentran allí las correlaciones entre las variables del estudio. 
- Work Engagement y Compromiso Laboral: ¿Es la edad un factor diferenciador? •

Tabla 1. Datos descriptivos de las variables del estudio

\begin{tabular}{|c|c|c|c|c|c|c|c|c|c|c|}
\hline & & $\alpha$ & $\mathrm{N}$ & Mín. & Máx. & $\mathrm{M}$ & $\mathrm{DE}$ & 1 & 2 & 3 \\
\hline 1 & C. Afectivo & 0.90 & 1594 & 0.00 & 4.00 & 3.60 & 0.48 & - & & \\
\hline 2 & C. Continuidad & $0.85^{*}$ & 5515 & 0.00 & 4.00 & 2.68 & 0.96 & $.359^{* *}$ & - & \\
\hline 3 & C. Normativo & 0.83 & 3751 & 0.00 & 4.00 & 3.12 & 0.75 & $.586^{*}$ & $.459^{* *}$ & - \\
\hline 4 & W. Engagement & 0.95 & 6303 & 1.00 & 5.00 & 4.47 & 0.48 & $.560^{* *}$ & $.288^{* *}$ & $.362^{* *}$ \\
\hline
\end{tabular}

N= Número total de datos, Mín: Mínimo, Máx: Máximo, M: Media DE: Desviación estándar. $\alpha=$ Alpha de Cronbach para el caso de C. Afectivo, C. Normativo y W. Engagement. Para C. Continuidad se calculó la confiabilidad compuesta.

Nota: Correlaciones: ${ }^{* *} \mathrm{p}<0.01$

Fuente: elaboración propia

La confiabilidad de la subescala de Compromiso de Continuidad fue baja al estimarla por el método Alpha de Cronbach (0.68). Sin embargo, dado que esta constaba de solamente cuatro ítems se hizo el cálculo a través del método de confiabilidad compuesta el cual arrojó un valor de 0.85 que se considera adecuado (Valdunciel, Flórez \& Miguel, 2008).

Todas las variables mostraron entre sí correlaciones estadísticamente significativas siendo las más fuertes de ellas las presentadas entre Compromiso Afectivo y Normativo, y entre Compromiso Afectivo y Work Engagement.

Las variables no se distribuyeron de forma normal (Compromiso Afectivo K-S=0.201, Compromiso de Continuidad K-S=0.117, Compromiso Normativo $\mathrm{K}-\mathrm{S}=0.127$, Work Engagement $\mathrm{K}-\mathrm{S}=0.135$ ) para todas las variables $p<0.001)$ lo que sustenta que los análisis deben realizarse con estadísticos no paramétricos.

Se utilizó la prueba estadística de Kruskal Wallis y se encontró que los rangos de edad establecidos sí permiten identificar diferencias estadísticamente significativas entre las variables. En la Tabla 2 se observan los resultados de la prueba.

Tabla 2. Prueba de Kruskal Wallis

\begin{tabular}{lcccc}
\hline & CA & CC & CN & EN \\
\hline Chi-cuadrado & 202.665 & 341.243 & 501.937 & 577.074 \\
Gl & 3 & 3 & 3 & 3 \\
Sig. Asintótica & .000 & .000 & .000 & .000 \\
\hline
\end{tabular}

CA: Compromiso Afectivo, CC: Compromiso de Continuidad, CN: Compromiso Normativo,

EN: Engagement. Gl: Grados de Libertad

Fuente: elaboración propia

Los descriptivos por edades se presentan en la Tabla 3. Allí también se resumen los resultados de la prueba no paramétrica con la que se compararon los grupos para identificar si existían diferencias estadísticamente significativas entre cada par de ellos y se presenta el tamaño del efecto. 
Tabla 3. U de Mann Withney y Descriptivos por grupos de Edad

\begin{tabular}{|c|c|c|c|c|c|c|c|c|c|c|}
\hline & & & & & 1 & & 2 & & 3 & \\
\hline & & & M & $\mathrm{DE}$ & $\begin{array}{l}\text { U de Mann- } \\
\text { Whitney }\end{array}$ & $d$ & $\begin{array}{l}\text { U de Mann- } \\
\text { Whitney }\end{array}$ & $d$ & $\begin{array}{l}\text { U de Mann- } \\
\text { Whitney }\end{array}$ & $d$ \\
\hline \multirow{4}{*}{1} & & CA & 3.54 & 0.5 & - & & & & & \\
\hline & De $18 \mathrm{a}$ & $\mathrm{CC}$ & 2.63 & 0.97 & - & & & & & \\
\hline & 25 años & $\mathrm{CN}$ & 2.95 & 0.77 & - & & & & & \\
\hline & & EN & 4.39 & 0.5 & - & & & & & \\
\hline & & CA & 3.55 & 0.5 & 4330859.5 & 0.021 & - & & & \\
\hline & De 26 a & CC & 2.52 & 0.99 & $4112843.5^{*}$ & 0.115 & - & & & \\
\hline & 35 años & $\mathrm{CN}$ & 2.97 & 0.8 & 4293365.5 & 0.015 & - & & & \\
\hline & & $\mathrm{EN}$ & 4.39 & 0.49 & 4023585.5 & 0.000 & - & & & \\
\hline \multirow{4}{*}{3} & & CA & 3.62 & 0.46 & $2737529.5^{*}$ & 0.003 & $9625439.5^{*}$ & 0.034 & - & \\
\hline & De 36 a & $\mathrm{CC}$ & 2.63 & 0.99 & 2981239.5 & 0.000 & $9664045.5^{*}$ & 0.106 & - & \\
\hline & 45 años & $\mathrm{CN}$ & 3.14 & 0.74 & $2531079.5^{*}$ & 0.128 & $9042127^{*}$ & 0.128 & - & \\
\hline & & $\mathrm{EN}$ & 4.48 & 0.46 & $2343920^{*}$ & 0.043 & $8833877.5^{*}$ & 0.043 & - & \\
\hline \multirow{4}{*}{4} & & CA & 3.66 & 0.45 & $4275586^{*}$ & 0.053 & $15047686.5^{*}$ & 0.019 & $11034625.5^{*}$ & 0.019 \\
\hline & 46 años & $\mathrm{CC}$ & 2.85 & 0.9 & $4369278.5^{*}$ & 0.229 & $14025730.5^{*}$ & 0.211 & $10291412.5^{*}$ & 0.211 \\
\hline & o más & $\mathrm{CN}$ & 3.27 & 0.67 & $3795489.5^{*}$ & 0.097 & $13665171^{*}$ & 0.097 & $10793458^{*}$ & 0.097 \\
\hline & & $\mathrm{EN}$ & 4.58 & 0.45 & $2887028^{*}$ & 0.048 & $10801327^{*}$ & 0.048 & $7930446^{*}$ & 0.048 \\
\hline
\end{tabular}

M: Media, DE: Desviación Estándar, $d$ : tamaño del efecto

En la mayoría de las variables el resultado se incrementa a medida que lo hace la edad. Solo para la categoría de Compromiso de Continuidad no fue posible identificar un patrón en tanto que la puntuación del grupo de personas más jóvenes disminuía en relación con el segundo grupo, se mantenía en el tercero, e incrementaba en el último.

De las 24 comparaciones posibles dos no mostraron diferencias y dos más no resultaron significativas a nivel estadístico. Todos estos casos correspondieron a comparaciones con el grupo de personas más jóvenes.

Pese a las diferenciadas halladas, el cálculo del tamaño del efecto mostró que ninguno de los casos correspondía a un tamaño mediano o grande (Coe \& Merino, 2003).

\section{Discusión}

La hipótesis inicial del estudio respecto a que la edad era una variable que podría explicar diferencias en el compromiso con la organización y con el trabajo fue corroborada con los datos de esta investigación. Esto es coherente con lo reportado en algunas otras investigaciones (Mathieu \& Zajac, 1990; Park \& Gursoy, 2012). Sin embargo, en los antecedentes revisados no se hacía alusión al cálculo del tamaño de la diferencia. Al aplicar dicho procedimiento a los datos se encontró que, pese a la significancia hallada, las distancias entre un grupo y otro eran muy sutiles. Es probable que el hecho de haber trabajado con una muestra grande facilitara encontrar las diferencias aun cuando estás representaban una variación mínima en la puntuación, es decir, cuando el cambio en la magnitud del atributo fuese pequeño. 
Llama la atención que el grupo más heterogéneo corresponde al de las personas más jóvenes, lo que señala que posiblemente es el más difícil de caracterizar. Las altas desviaciones encontradas en este grupo señalan que los encuestados no coinciden entre sí en las formas de responder a los cuestionarios y que por tanto sus formas de comprometerse con la organización y de engancharse con la tarea son bastante disímiles.

Aunque explicar estos resultados requiere de otros análisis y de la inclusión de más variables, gracias a la revisión de literatura científica y a la experiencia acumulada en el Centro de Investigación, es factible plantear algunas posibles explicaciones a las variaciones encontradas. Sin embargo, será necesario tener presente que, dado el tamaño de la diferencia, es probable que existan muchas otras variables diferentes a la edad con mayor capacidad de explicar las variaciones en el compromiso.

\section{Compromiso Afectivo y Edad}

Se encontraron diferencias en el Compromiso Afectivo a medida que aumentaba la edad, no obstante estas variaciones solo se tornaron significativas a partir de los 35 años. Tal y como se mencionó, la diferencia es mínima.

Uno de los elementos fundamentales para que se genere compromiso con la organización es la coincidencia entre los valores de esta y los de la persona (Boxx, Odom \& Dunn, 1991, Edwards \& Cable, 2009). Puede existir una dificultad por parte de las generaciones más jóvenes para hacer coincidir los valores propios con los que promueve la empresa (Hershatter \& Epstein, 2010). Mientras que esta última privilegia aspectos como la estabilidad laboral, la sociedad actual, mediada por el impacto de los avances tecnológicos, promueve el cambio y no incluye la estabilidad como un aspecto inherente al contrato psicológico establecido con la organización (Thompson \& Gregory, 2012). De la misma manera, mientras que las generaciones pasadas privilegiaban el conformismo y la abnegación, las más recientes se caracterizan por ser ambiciosas lo cual puede ser leído en las compañías como una falta de adecuación a la cultura organizacional (Thompson \& Gregory, 2012).

Las generaciones más recientes, dado el impacto del desarrollo tecnológico, se caracterizan por ejecutar diversas tareas de forma simultánea (Carrier, Cheever, Rosen, Benitez \& Chang, 2009), lo que podría entrar en conflicto con el modelo tradicional de las empresas que promueven un estilo de trabajo donde el valor del mismo se centra en funciones y actividades específicas. Aunque al personal mayor se le presenta como una barrera el dominio de las herramientas tecnológicas (Juste, Carballo, \& López, 2015), es probable que la focalización en una sola actividad le permita ajustarse más fácilmente a las estructuras y normas organizacionales tradicionales.

La generación de personas más jóvenes contempla como ejes centrales de su futuro profesional, por un lado, un conjunto de valores diferentes de corte más individualista y exigente $\mathrm{y}$, por otro, una visión del mercado de trabajo caracterizada por menores grados de regulación (Simon, 2007).

\section{Compromiso Normativo y Edad}

En tanto que el Compromiso Normativo se caracteriza por una sensación de deuda moral con respecto a la organización y por el "deber" más que por el "querer", se puede hallar en la transformación de los valores de la sociedad una explicación. Es preciso comprender que anteriormente se promovía la lealtad y la permanencia y que, por tanto, quienes hoy son mayores fueron educados bajo ese modelo (Ardila, 1986). Lo anterior podría explicar que las personas de mayor edad evidencien un compromiso normativo más elevado que los más jóvenes.

Muchas de las personas que en la actualidad han cumplido recientemente su edad de jubilación 
o se encuentran cercanas a la misma, se niegan a retirarse de la labor productiva que han desempeñado durante muchos años (Tacchino, 2013). El hecho de que busquen la forma de mantenerse vinculados con el trabajo es evidencia de la adaptación que dicha generación muestra con el sistema laboral tradicional. Estas personas se muestran más orientadas a establecerse y tienen una menor disposición a reubicarse o abandonar la organización con propósitos de promoción. Por el contrario, los jóvenes están insertos evolutivamente en una etapa de cambio y lo asumen de forma mucho más natural (Segura \& García, 2010).

El carácter de deuda que acompaña al Compromiso Normativo suele requerir de un tiempo prolongado para que se establezca la relación de lealtad entre la persona y la organización (Khattaki \& Sethi, 2012). La poca fuerza de este componente en el personal más joven podría estar también explicada por un corto tiempo de antigüedad en la empresa y por los elevados intereses de movilidad y cambio de dicha generación.

\section{Compromiso de Continuidad y Edad}

El Compromiso de Continuidad se genera a partir del balance que la persona realiza al comparar los costos y beneficios de permanecer en la organización. Como se mencionó en los resultados, en dicha categoría no fue posible identificar un patrón, sin embargo, llama la atención que la puntuación más alta aparece en el grupo de las personas más adultas. Para el análisis es preciso tener en cuenta que a mayor tiempo en una organización es probable que se haya hecho un mayor número de inversiones y que por tanto retirarse represente una pérdida más elevada.

Sumado a ello, el tema de la discriminación etaria, es decir, de una actitud reticente por parte de las organizaciones hacia la posibilidad de contratar personas mayores (Nazar \& Figueroa, 2015), ha ocasionado que a más edad se tengan menos probabilidades de insertarse en el mundo laboral
(Galiani \& Hopenhayn, 2000). Dicho asunto hace que este grupo poblacional valore mucho más el vínculo que ya tiene con la organización.

El Compromiso de Continuidad no solo se gesta a partir de lo que la organización le da a la persona, sino también a partir de lo que el mercado laboral no le brinda (Iverson \& Buttigieg, 1999). Una persona se puede quedar en una empresa porque le proporciona muchos beneficios o, porque a pesar de lo desfavorable que puede ser permanecer allí, la oferta externa sería aún menos conveniente. En este sentido, las personas mayores temen más al desempleo y eso incrementa su compromiso de continuidad.

\section{Work Engagement y Edad}

Una de las posibles explicaciones de las diferencias en el nivel de Work Engagement en los diversos grupos de edad corresponde a la capacidad que tiene el trabajador de moldear la tarea según sus preferencias (Tims, Bakker, \& Derks, 2012). Se parte del supuesto de que la edad está asociada con el conocimiento y la experticia en la labor. Aunque ello no necesariamente implica un mejor desempeño, sí se asocia con una mayor capacidad de ajustar el trabajo a las propias preferencias aumentando el nivel de desafíos respecto a los cuales los trabajadores tienen buenos niveles de autoeficacia, y disminuyendo las demandas que creen exceden su capacidad de respuesta. Lo anterior tiene correlaciones positivas con el Work Engagement (Tims, Bakker, \& Derks, 2013). Se concluye entonces que quien lleva más tiempo en la vida desempeñando una misma tarea es más hábil para negociar sus responsabilidades y hacerse cargo de los procesos que le atraen.

La habilidad es uno de los predictores importantes del desempeño (Toro, 1992) y se desarrolla a lo largo del tiempo y solo con práctica. Favorece la ejecución exitosa de las actividades, lo cual es una de las mejores fuentes de autoeficacia (Olaz, 2004). A su vez la autoeficacia correlaciona positivamente con Work Engagement (Del Líbano, 
Llorens, Salanova, \& Schaufeli 2012). Esto sugiere que el Work Engagement puede aumentar gracias al aumento de la autoeficacia producido por el desarrollo de la habilidad producto de años de práctica y aplicación.

\section{Limitaciones}

Aunque los hallazgos pueden resultar de interés para la comunidad académica y empresarial, el estudio presenta algunas limitaciones que se señalan a continuación:

La muestra fue seleccionada por disponibilidad y no de manera probabilística. No obstante, el número de participantes y de empresas involucradas otorgan cierto nivel de representatividad a los resultados.

Los análisis de Work Engagement no se pudieron realizar teniendo en cuenta cada una de las dimensiones de la variable pues el instrumento empleado para la medición del constructo no reportaba dicha estructura factorial, sino que arrojaba un único factor. Sin embargo, es un instrumento que muestra indicadores psicométricos adecuados.

Para futuras investigaciones sería interesante realizar análisis teniendo en cuenta otras variables del estudio como la empresa, el tamaño de la misma o la antigüedad de los participantes. Se sugiere también realizar un estudio longitudinal. Infortunadamente no es posible con los datos de esta investigación pues las bases de datos no cuentan con códigos de identificación de cada uno de los encuestados.

\section{Conclusiones}

Las diferencias generacionales en los valores del trabajo pueden ocasionar cambios en la forma en la cual las personas se comprometen con el mismo (Park, \& Gursoy, 2012). Los jóvenes, si bien son los más solicitados por las empresas, suponen un reto para éstas en términos de su vinculación afectiva y permanencia. Paradójicamente las personas mayores, hacia las que se evidencia una actitud reticente por parte de los empleadores para contratarlos, desarrollan mayores niveles de Compromiso Laboral y Work Engagement. Una realidad que sugiere una importante reflexión.

Pese a lo anterior, dado que las diferencias son pequeñas, gestionar el compromiso requiere más de una comprensión de los antecedentes de dicho fenómeno que de una intervención centrada en la edad.

\section{Bibliografía}

Almeida, M., \& Fierro, I. (2015). Comportamiento organizacional positivo: las implicaciones del engagement en el entorno laboral. Eca Sinergia. Revista Digital, 6(2), 150-164.

Allen, N. \& Meyer, J. (1990). The measurement and antecedents of affective, continuance and normative commitment to the organization. Journal of Occupational Psychology, 63(1), 1-18. http://dx.doi. org/10.1111/j.2044-8325.1990.tb00506.x.

Ardila, R. (1986). Psicologia del hombre colombiano: cultura y comportamiento social. Bogotá. Planeta.

Boxx, W., Odom, R., \& Dunn, M. (1991). Organizational Values and Value Congruency and Their Impact on Satisfaction, Commitment, and Cohesion: An Empirical Examination within the Public Sector. Public Personnel Management, 20(2), 195-205. http://dx.doi.org/10.1177/009102609102000207.

Calderón G., Naranjo J.., \& Álvarez, C. (2010). Gestión humana en la empresa colombiana: sus características, retos y aportes. Una aproximación a un sistema integral. Cuadernos de Administración, 23(41), 13-36.

Carrier, L., Cheever, N., Rosen, L., Benitez, S., \& Chang, J. (2009). Multitasking across generations: Multitasking choices and difficulty ratings in three generations of Americans. Computers In Human Behavior, 25(2), 483-489. http://dx.doi. org/10.1016/j.chb.2008.10.012.

Castrejón, M. (2016). Relación entre los niveles de engagement y compromiso organizacional en personal de una institución de educación superior de la ciudad de Cajamarca. Tendencias en psicología, 2(2), 45-52.

Coe, R. \& Merino, C. (2003). Magnitud del Efecto: Una guía para investigadores y usuarios. Revista de Psicología de la PUCP, 21 (1), 147-177. 
Cincel S.A.S. (2015) Ficha técnica del Instrumento Nexos. Documento inédito.

Chirinos, N. (2009). Características generacionales y los valores. Su impacto en lo laboral. Observatorio Laboral Revista Venezolana, 2(4), 6.

Edwards, Jeffrey R.; Cable, Daniel M (2009). The value of value congruence. Journal of Applied Psychology, 94(3), 654-677.

Galiani, S., \& Hopenhayn, H. (2000). Duración y riesgo de desempleo en Argentina, series Mercado de Trabajo y Relaciones Industriales. Buenos Aires, Fundación Argentina para el Desarrollo con Equidad (FADE), inédito.

Hershatter, A. \& Epstein, M. (2010). Millennials and the World of Work: An Organization and Management Perspective. J Bus Psychol, 25(2), 211-223. http:// dx.doi.org/10.1007/s10869-010-9160-y.

Irizarry-Hernández, E. B., \& de Arecibo (2009). La generación $\mathrm{Y}$ o generación milenaria: el nuevo paradigma laboral. Revista Empresarial Inter Metro / Inter Metro Business Journal Fall 2009, 5 (2), 10.

Iverson, R. \& Buttigieg, D. (1999). Affective, Normative and Continuance Commitment: Can the 'Right Kind' of Commitment be Managed?. Journal of Management Studies, 36(3), 307-333. http://dx.doi. org/10.1111/1467-6486.00138.

Juste, M. R. P., Carballo, J. G. S., \& López, B. R. (2015). Las personas mayores y las TIC: un compromiso para reducir la brecha digital. Pedagogía Social, 26, 337.

Khattaki, A. A., \& Sethi, S. (2012). Organization Normative Commitment (ONC) has Psychological Positive Effects on Employees' Performance. Abasyn Journal of Social Sciences, 5(1), 99-110.

Líbano del, M., Llorens, S., Salanova, M., \& Schaufeli, W. (2012). About the Dark and Bright Sides of Selfefficacy: Workaholism and Work Engagement. Span. J. Psychol., 15(02), 688-701. http://dx.doi. org/10.5209/rev_sjop.2012.v15.n2.38883.

Littlewood,H.(2003). Metanálisis del Apoyo Organizacional Percibido y Compromiso Organizacional en Organizaciones Mexicanas. Revista Interamericana de Psicología Ocupacional, 22 (2), 45-56.

López, M., García,S., Pando, M.(2015).Factores Psicosociales y Compromiso organizacional (Work Engagement) en trabajadores del campo. Revista Iberoamericana de Contaduria, Economía y Administración: RICEA, 4(7), 1-1.
Lowe, D., Levitt, K., \& Wilson, T. (2011). Solutions for retaining generation $\mathrm{Y}$ employees in the workplace. IEEE Engineering Management Review, 39(2), 4652. http://dx.doi.org/10.1109/emr.2011.5876174.

Mathieu, J. \& Zajac, D. (1990). A review and meta-analysis of the antecedents, correlates, and consequences of organizational commitment. Psychological Bulletin, $108(2), 171-194$.

Meyer, J. (2014) Employee Commitment, Motivation, 3 and Engagement: Exploring the Links. En Gagné, M. (Ed.), The Oxford handbook of work engagement, motivation, and self-determination theory. USA: Oxford University Press,

Meyer, J., Stanley, D., Herscovitch, L., \& Topolnytsky, L. (2002). Affective, Continuance, and Normative Commitment to the Organization: A Meta-analysis of Antecedents, Correlates, and Consequences. Journal Of Vocational Behavior, 61(1), 20-52. http:// dx.doi.org/10.1006/jvbe.2001.1842.

Meyer, J. \& Allen, N. (1997). Commitment in the workplace. Thousand Oaks, Calif.: Sage Publications.

Mowday, R. T., Steers, R. M., \& Porter, L. W. (1979). The measurement of organizational commitment. Journal of vocational behavior, 14(2), 224-247.

Nazar, G., \& Figueroa, C. A. (2015). Creencias estereotípicas sobre el desempeño laboral de trabajadores mayores en Chile. Psicoperspectivas, 14(1), 114-125.

Olaz, F. (2004). Aportes del meta-análisis a la teoría de la autoeficacia para el desarrollo de carrera. Estudios De Psicología, 25(1), 57-72. http://dx.doi. org/10.1174/021093904773487015.

Ogg, J., \& Bonvalet, C. (2006). The Babyboomer generation and the birth cohort of 1945-1954: a European perspective. Artículo presentado en el seminario organizado por Cultures of Consumption Research Programme.

Park, J. \& Gursoy, D. (2012). Generation effects on work engagement among U.S. hotel employees. International Journal Of Hospitality Management, 31(4), 1195-1202. http://dx.doi.org/10.1016/j. ijhm.2012.02.007.

Ryder, N. (1965). The Cohort as a Concept in the Study of Social Change. American Sociological Review, 30(6), 843. http://dx.doi.org/10.2307/2090964

Salanova, M.; Schaufeli, W.B.; Llorens, S.; Peiró, J.M. y Grau, R. (2000). Desde el "burnout" al "engagement": ¿una nueva perspectiva? Revista de Psicología del Trabajo y de las Organizaciones, 16(2), 117-134. 
Salanova, M. \& Schaufeli, W. (2009). El engagement en el trabajo. Madrid: Alianza Editorial.

Segura, E. R., \& García, M. A. P. (2010). La convivencia de diferentes generaciones en la empresa; Compatibilización y liderazgo integral. In 4th International Conference On Industrial Engineering and Industrial Management (pp. 160-170).

Simon, C., \& Allard, G. (2007). Generación y mercado laboral: modelos de gestión de recursos humanos para los jóvenes profesionales. Aedipe: Revista de la Asociación Española de Dirección de Personal, (45), 58-61.

Tacchino, J. I. (2013). Will Baby Boomers Phase into Retirement? Journal of Financial Service Professionals, 67(3).

Thompson, C. \& Gregory, J. (2012). Managing Millennials: A framework for improving attraction, motivation, and retention. The Psychologist-Manager Journal, 15(4), 237-246. http://dx.doi.org/10.1080/108871 56.2012 .730444

Tims, M., Bakker, A., \& Derks, D. (2012). Development and validation of the job crafting scale. Journal of
Vocational Behavior, 80(1), 173-186. http://dx.doi. org/10.1016/j.jvb.2011.05.009.

Tims, M., Bakker, A., \& Derks, D. (2013). The impact of job crafting on job demands, job resources, and wellbeing. Journal Of Occupational Health Psychology, 18(2), 230-240. http://dx.doi.org/10.1037/ a0032141.

Toro, F. (1992b). Desempeño y productividad. Medellín: Cincel.

Valdivieso, C. U. (2002). Psicología del ciclo vital: hacia una visión comprehensiva de la vida humana. Revista Latinoamericana de Psicología, 34(1-2), 17-27.

Valdunciel, L., Flórez, M., y Miguel, J. Á. (2008). Análisis de la calidad del servicio que prestan las entidades bancarias y su repercusión en la satisfacción del cliente. Revista Asturiana de Economía, 38, 79-107.

Zager, G. (2015) Disentangling the Overlap Between Employee Engagement and Passion. Psibologijske teme 24, No. 2 (2015): 233-258. 